\title{
Antifungal susceptibility and phenotypic virulence markers of Candida species isolated from Nepal
}

\author{
Supram Hosuru Subramanya ${ }^{\text {* }} \mathbb{0}$, Bharat Prasad Baral ${ }^{1}$, Nawal Kishor Sharan ${ }^{1}$, Niranjan Nayak', Yang Metok', \\ Brijesh Sathian", Indira Bairy ${ }^{2}$ and Shishir Gokhale ${ }^{1}$
}

\begin{abstract}
Objective: Candida species are part of the commensal microflora in many anatomical sites of the human body; however, breach in the integrity of the body part and impaired immunity of the host can lead to invasive candidiasis. A number of virulence determinants could contribute towards its pathogenicity. Thus we attempted to evaluate the in vitro expression of different virulence factors among clinical isolates of Candida species and assayed their susceptibility patterns against a range of antifungal agents.

Result: Of the total of 71 isolates we obtained, 48 (67.6\%) were Candida albicans, 11 (15.49\%) Candida tropicalis, 09 (12.67\%) Candida glabrata and 03 (4.22\%) were Candida krusei. Proteinase, phospholipase and esterase production could be revealed amongst $43(60.56 \%), 44$ (61.97\%) and 49 (69.01\%) isolates respectively. None of the isolates showed DNAase activity. Fifty-five (77.39\%) isolates were biofilm producers, and 53 (74.6\%) exhibited high cell surface hydrophobicity.
\end{abstract}

Keywords: Candida species, Virulence factors, Antifungal susceptibility testing

\section{Introduction}

Candida species are part of the commensal microflora in many anatomical sites of the human body [1]. If host immunity is compromised, or there is disruption in the skin or mucosal site where Candida remains as a commensal, there is always a chance for Candida to invade and cause a wide range of infections with significant morbidity and mortality [2,3]. Though Candida albicans has been associated with most human infections, there has been increasing reports of infections due to non-albicans Candida species in the recent past $[4,5]$. A number of virulence attributes such as biofilm formation, proteinase, esterase, phospholipase activities and drug resistance contributing towards the pathogenicity of Candida have been proposed. Thus our study was conducted to determine and compare in vitro production of virulence factors by Candida species and their antifungal

\footnotetext{
*Correspondence: supram.gowda@gmail.com

${ }^{1}$ Manipal College of Medical Sciences, Pokhara, Nepal

Full list of author information is available at the end of the article
}

susceptibility patterns. To the best of our knowledge, the present study is the first of its kind in Nepal.

\section{Main text \\ Materials and methods \\ Candida isolates}

A total of 71 Candida species isolated between 2014 and 2016 from various clinical samples (Additional file 1: Table S1) were studied. Organisms were identified by the standard laboratory techniques [6], and growth on HiChrome candida differential agar (Hi-Media, India).

\section{Preparation of yeast suspension for enzymatic activity}

A loopful of the culture was streaked onto Sabouraud's dextrose agar (SDA) with chloramphenicol $(0.05 \mathrm{~g} / \mathrm{l}$; HiMedia, India) and incubated at $37{ }^{\circ} \mathrm{C}$ for $24-48 \mathrm{~h}$. Cells were harvested and suspended in sterile PBS and matched to $0.5 \mathrm{Mc}$ Farland. The final suspension was adjusted to contain $2.5 \times 10^{6}$ yeast cells $/ \mathrm{ml}$. The above inocula were used for all enzymatic studies, as well as for 
the biofilm activity. Qualities of all assays were checked using known positive and negative controls.

\section{Biofilm formation}

The method standardised by Malek et al. [6] was followed to develop biofilms in 96 well microtiter plates. Measurement of biofilm mass by quantitative method was performed using crystal violet for staining the biomass and metabolic activity of the biofilm cells was assessed colorimetrically based on reduction of sodium 39-[1-(phenylamino-carbonyl)-3,4-tetrazolium]-bis(4methoxy-6-nitro)benzene sulfonic acid hydrate (XTT) as described elsewhere [7]. Biomass was also demonstrated by fluorescent microscopy with calcofluor white staining (Fig. 1). Known biofilm producer and non-biofilm producer Candida strains served as positive and negative controls respectively.

\section{Proteinase and phospholipase activities}

Proteinase activity was assessed by bovine serum albumin (BSA) agar based assay as described previously [8]. The presence of halo surrounding the growth representing proteinase activity was observed by staining with amido black. The egg yolk agar method as described earlier was employed for determining the phospholipase activity [9]. Pz (precipitation zone) values for both the tests were calculated according the parameters noted earlier $[8,9]$. Known proteinase and phospholipase positive and negative Candida strains served as controls.

\section{Esterase, deoxyribonuclease and cell surface hydrophobicity}

Esterase activity was noted in Tween-80 agar as described previously [10] and test for cell surface hydrophobicity $(\mathrm{CSH})$ was performed in accordance with the earlier devised technique [11]. DNase production was measured according to the standard protocol [11], using ATCC 25923 standard strain of Staphylococcus aureus as positive control. Known Esterase and CSH positive and negative Candida strains served as controls.

\section{Antifungal susceptibility testing}

All 71 isolates were subjected to antifungal susceptibility testing against amphotericin $\mathrm{B}$, voriconazole, fluconazole and caspofungin by microbroth dilution method based on the Clinical and Laboratory Standards Institute M27-A3 standard [12]. Tests were interpreted by visual method with the help of reading mirror after $24 \mathrm{~h}$ of incubation at $37{ }^{\circ} \mathrm{C}$. Candida parapsilosis ATCC 95142 and C. albicans ATCC 90028 were used as controls. Antifungal compounds were obtained as pure powders from the manufacturer, Sigma-Aldrich Laborchemikalien $\mathrm{GmbH}$, Germany.

\section{Statistical analysis}

Descriptive statistics were used for analyzing the data entered in Microsoft Excel 2010 by Statistical Analysis System (SAS) and Origin Pro 2016. MIC values of different antifungal agents against $C$. albicans and non albicans Candida were expressed in terms of range, median and
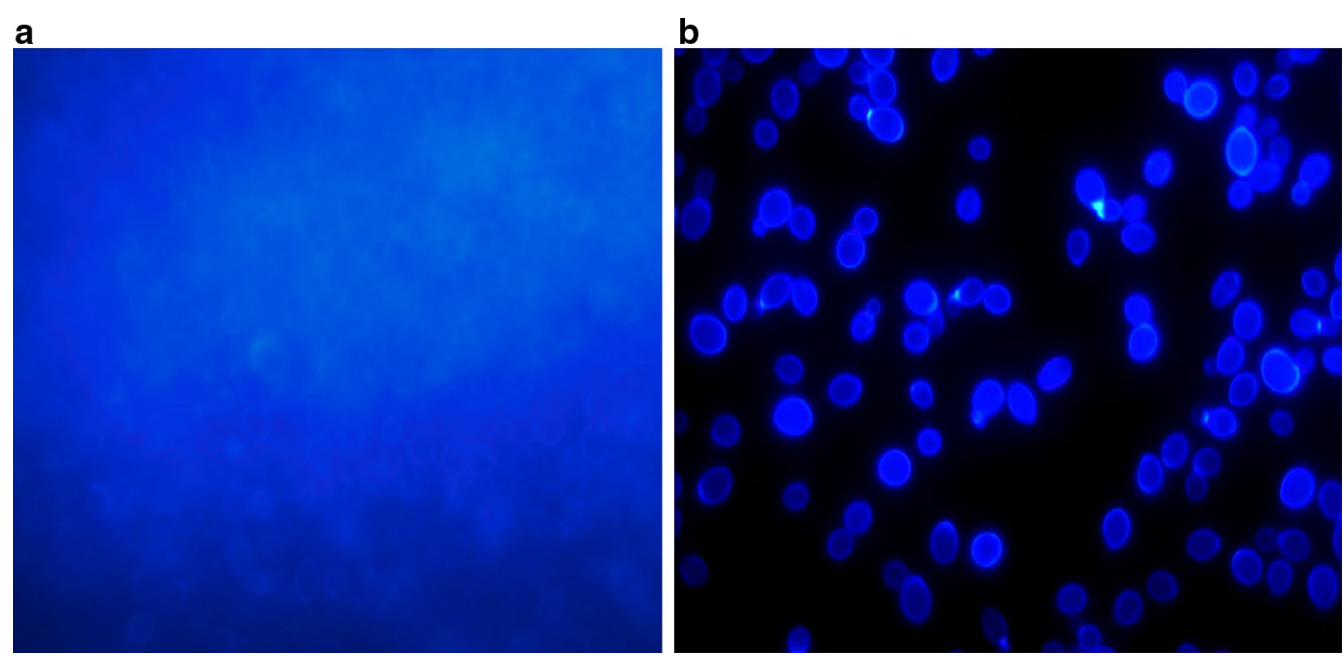

Fig. 1 Demonstration of biomass of biofilm by fluorescent microscopy using calcofluor white stain: Calcofluor staining of biofilm produced in the glass slide showing (a) hazy appearance which was due to diffuse staining of the extracellular material (cell-wall-like polysaccharides). Blastospore communities were covered by the matrix (rarely seen) and (b) Planktonic cells of Candida albicans showing oval budding blastospores (magnification $\times 1000)$ 
geometric mean. Variations among MIC values of antifungal agents against biofilm producing and non-biofilm producing Candida albicans and non-albicans Candida species were assessed by using minimum, maximum, median, and 90th percentile and box plot.

\section{Results}

Out of a total of 71 Candida isolates, 48 (67.6\%) were C. albicans; 11 (15.5\%) were C. tropicalis; 9 (12.7\%) C. glabrata and 3 (4.2\%) were C. krusei. As depicted in Table 1, proteinase, phospholipase and esterase activity could be detected amongst $43(60.6 \%), 44(62 \%)$, and $49(69 \%)$ of the isolates respectively. None of the isolates produced DNase. CSH, was observed among 54 (76\%) of the 71 isolates. As many as $55(77.4 \%)$ out of the total 71 isolates were found to be biofilm producers as evidenced by metabolic activity and biomass production (Fig. 1). Majority, i.e. $40(74.07 \%)$ of the 54 having high cell surface hydrophobicity produced biofilms. $36(75 \%)$ out of 48 C. albicans strains produced proteinase in contrast to only 7 (30\%) of the 23 non-albicans Candida species. Similarly, higher numbers of $C$. albicans strains were found to be phospholipase and esterase producers as compared to non albicans Candida (Table 1). Isolation rates of C. albicans from blood and indwelling devices were found to be much higher as compared to non albicans Candida species. Similarly biofilm production was seen among $84-100 \%$ of the blood and device isolates (Additional file 1: Table S1).

Tables 2 and 3 denote the antifungal susceptibility patterns of the isolates. Isolates were classified as sensitive, intermediately sensitive and resistant to each antifungal agent in accordance with the break point criteria laid down by CLSI [10]. During data analysis, both sensitive and intermediately sensitive isolates were categorized as one group, i.e. sensitive. As high as $97.9 \%$ (47/48), 85.4\% (41/48) and $77 \%(37 / 48)$ C. albicans isolates were sensitive to amphotericin $\mathrm{B}$, caspofungin and voriconazole respectively. Overall, 95.7\% (22/23) of non-albicans strains were found to be susceptible to amphotericin B and caspofungin. Amongst C. tropicalis all 11, i.e. $100 \%$ were sensitive to amphotericin B and caspofungin (Table 3). Fluconazole sensitivity of $C$. albicans, $C$. tropicalis and C. krusei ranged between 33.3 and $52 \%$. A total of $82.6 \%$ (19/23) of the non-albicans Candida were sensitive to voriconazole, only $56.5 \%(13 / 23)$ were sensitive to fluconazole.

Median MICs and geometric mean MIC (GMM) values of fluconazole were found much higher as compared to

Table 1 Virulence factors found among different Candida species

\begin{tabular}{|c|c|c|c|c|c|}
\hline & \multirow[t]{2}{*}{ Pz value } & \multirow{2}{*}{$\begin{array}{l}\text { Candida albicans } \\
(\mathrm{n}=48)\end{array}$} & \multicolumn{3}{|c|}{ Non albicans Candida species } \\
\hline & & & Candida tropicalis $(n=11)$ & Candida glabrata $(\mathrm{n}=9)$ & Candida krusei $(\mathrm{n}=3)$ \\
\hline \multicolumn{6}{|c|}{ Proteinase test } \\
\hline Strong & $<0.79$ & 35 & 5 & 1 & 0 \\
\hline Mild & $0.80-0.89$ & 1 & 1 & 0 & 0 \\
\hline Weak & $0.90-0.99$ & 0 & 0 & 0 & 0 \\
\hline Negative & $=1$ & 12 & 5 & 8 & 3 \\
\hline \multicolumn{6}{|c|}{ Phospholipase test } \\
\hline Strong & $<0.79$ & 17 & 2 & 1 & 0 \\
\hline Mild & $0.80-0.89$ & 8 & 0 & 0 & 0 \\
\hline Weak & $0.90-0.99$ & 10 & 3 & 2 & 1 \\
\hline Negative & $=1$ & 13 & 6 & 6 & 2 \\
\hline \multicolumn{6}{|l|}{ Esterase test } \\
\hline Strong & $<0.79$ & 31 & 8 & 3 & 1 \\
\hline Mild & $0.80-0.89$ & 6 & 0 & 0 & 0 \\
\hline Weak & $0.90-0.99$ & 0 & 0 & 0 & 0 \\
\hline Negative & $=1$ & 11 & 3 & 6 & 2 \\
\hline \multicolumn{6}{|c|}{ Biofilm production } \\
\hline Positive & - & 36 & 10 & 6 & 3 \\
\hline Negative & - & 12 & 1 & 3 & 0 \\
\hline \multicolumn{6}{|c|}{ Cell surface hydrophobicity (\%) } \\
\hline Strong & $>20$ & 35 & 10 & 7 & 2 \\
\hline Mild & 10-19.99 & 9 & 1 & 2 & 1 \\
\hline Weak & $0.1-9.99$ & 4 & 0 & 0 & 0 \\
\hline Negative & $<0.1$ & 0 & 0 & 0 & 0 \\
\hline
\end{tabular}


Table 2 In vitro antifungal susceptibility profile of various Candida species

\begin{tabular}{|c|c|c|c|c|c|c|c|c|c|c|c|c|}
\hline \multirow[t]{3}{*}{ CLSI breakpoints $(\mu \mathrm{g} / \mathrm{ml})$} & \multicolumn{3}{|c|}{ Amphotericin B } & \multicolumn{3}{|c|}{ Caspofungin } & \multicolumn{3}{|c|}{ Fluconazole } & \multicolumn{3}{|c|}{ Voriconazole } \\
\hline & $\mathrm{S}$ & I & $\mathbf{R}$ & $S$ & 1 & $\mathbf{R}$ & $S$ & I & $\mathbf{R}$ & $S$ & I & $\mathbf{R}$ \\
\hline & $\leq 4$ & 8-16 & $\geq 32$ & $\leq 0.25$ & 0.5 & $\geq 1$ & $\leq 2$ & 4 & $\geq 8$ & $\leq 0.12$ & $0.25-0.5$ & $\geq 1$ \\
\hline C. albicans, $n=48(\%)$ & $47(97.9)$ & $0(0)$ & $1(2.1)$ & $41(85.4)$ & $4(8.3)$ & $3(6.3)$ & $11(23)$ & $14(29)$ & $23(48)$ & $37(77)$ & $11(23)$ & $0(0)$ \\
\hline C. tropicalis, $\mathrm{n}=11(\%)$ & $0(0)$ & $11(100)$ & $0(0)$ & $11(100)$ & 00 & 00 & $4(36.4)$ & $1(9)$ & $6(54.6)$ & 00 & $9(81.8)$ & $0(18.2)$ \\
\hline C. glabrata, $n=9(\%)$ & $0(0)$ & $9(100)$ & $0(0)$ & $9(100)$ & 00 & 00 & $6(66.7)$ & $1(11.1)$ & $2(22.2)$ & 00 & $8(88.9)$ & $1(11.1)$ \\
\hline C. krusei, $\mathrm{n}=3(\%)$ & 00 & $2(66.7)$ & $1(33.3)$ & $2(66.7)$ & $1(33.3)$ & $0(0)$ & $1(33.3)$ & $0(0)$ & $2(66.7)$ & 00 & $2(66.7)$ & $1(33.3)$ \\
\hline
\end{tabular}

Table 3 MIC values of different antifungal agents against C. albicans and non albicans Candida

\begin{tabular}{|c|c|c|c|c|c|c|c|c|c|c|c|c|}
\hline \multirow[t]{2}{*}{ Candida isolates } & \multicolumn{3}{|c|}{ Fluconazole MIC } & \multicolumn{3}{|c|}{ Amphotericin B MIC } & \multicolumn{3}{|c|}{ Voriconazole MIC } & \multicolumn{3}{|c|}{ Caspofungin MIC } \\
\hline & Range & Median & GM & Range & Median & GM & Range & Median & GM & Range & Median & GM \\
\hline C. albicans & $0.125-64$ & 4 & 5.039 & $0.031-32$ & 0.25 & 0.3 & $0.031-0.5$ & 0.125 & 0.1 & $0.031-2$ & 0.0625 & 0.068 \\
\hline Non albicans Candida & $0.0313-64$ & 4 & 3.718 & $0.0625-32$ & 0.5 & 0.417 & $0.031-0.5$ & 0.0625 & 0.077 & $0.031-0.5$ & 0.0625 & 0.0625 \\
\hline
\end{tabular}

those for amphotericin B, caspofungin and voriconazole both for C. albicans and non albicans Candida (Table 3). While the median MICs and GMM values were found to be the lowest with respect to caspofungin in both $C$. albicans $(0.00625$ and $0.068 \mu \mathrm{g} / \mathrm{ml})$ and non-albicans Candida $(0.0625$ and $0.0628 \mu \mathrm{g} / \mathrm{ml})$ species, those for voriconazole were 0.125 and $0.1 \mu \mathrm{g} / \mathrm{ml}$ respectively against $C$. albicans and 0.0625 and $0.077 \mu \mathrm{g} / \mathrm{ml}$ respectively against non-albicans Candida.

Based upon the median MIC data, we determined the number of strains showing high MIC values (higher than the median MIC) and those exhibiting low MIC values (lower than the median MIC), in order to see if there was any correlation between biofilm production and drug resistance. A significant difference could be noted amongst the non-albicans Candida, against amphotericin $\mathrm{B}$, fluconazole and caspofungin. As depicted in Fig. 2, a large number of the non-biofilm producing $C$. albicans strains showed high MIC values against amphotericin B, fluconazole and voriconazole. Similarly, non-albicans Candida that were non-biofilm producers exhibited moderately higher MICs against amphotericin B.

\section{Discussion}

Many invasive Candida infections are attributable to some of the potential virulence factors of the organism such as proteinase, phospholipase and esterase. Majority $(72-77 \%)$ of the $C$. albicans strains in this study were capable of producing proteinase, phospholipase and esterase. A high rate of phospholipase (94.7\%) and a moderately high rate of proteinase $(73.7 \%)$ production amongst $C$. albicans clinical isolates were reported earlier [13-15]. Proteinase as a major virulence determinant of both $C$. albicans and non albicans Candida in invasive infections was documented earlier [16]. Gokee et al. [17] detected proteinase in $89.7 \%$ of C. albicans isolates, and only in $25.8 \%$ of the non-albicans isolates. Inci et al. [18] reported that 95\% C. albicans and $24 \%$ non-albicans Candida were proteinase producers. We noted proteinase production among $75 \%$ of our C. albicans isolates and only $30 \%$ of the non-albicans isolates.

The role of esterase in the pathogenesis of invasive candidiasis is debatable [14-16]. However, earlier studies [18] demonstrated that both C. albicans and non albicans Candida species showed esterase activity. In our study, esterase was detected amongst $77 \%$ of $C$. albicans isolates as compared to $52 \%$ non-albicans isolates, difference being marginally higher among the $C$. albicans isolates. Tellapragada et al. [19] found no significant difference in the esterase activities among invasive and non-invasive Candida. They did not, however, compare this observation between $C$. albicans and non albicans isolates.

In the present study, resistance rates for the azoles were substantially higher as compared to amphotericin B or caspofungin, especially in C. tropicalis (Table 2). Additionally MIC ranges, median MICs and geometric mean titres were higher for fluconazole both for $C$. albicans and non-albicans Candida (Table 3). These findings were similar to those reported by others [20], who proposed that azole resistance in Candida was of concern because azoles like fluconazole happened to be the most common antifungal agent used for the treatment and prophylaxis of candidiasis in organ transplant recipients. In yet another study [5], nosocomial isolates of C. albicans were shown to have far lower sensitivity rates towards fluconazole. Seneviratne et al. [21] very recently reported that 


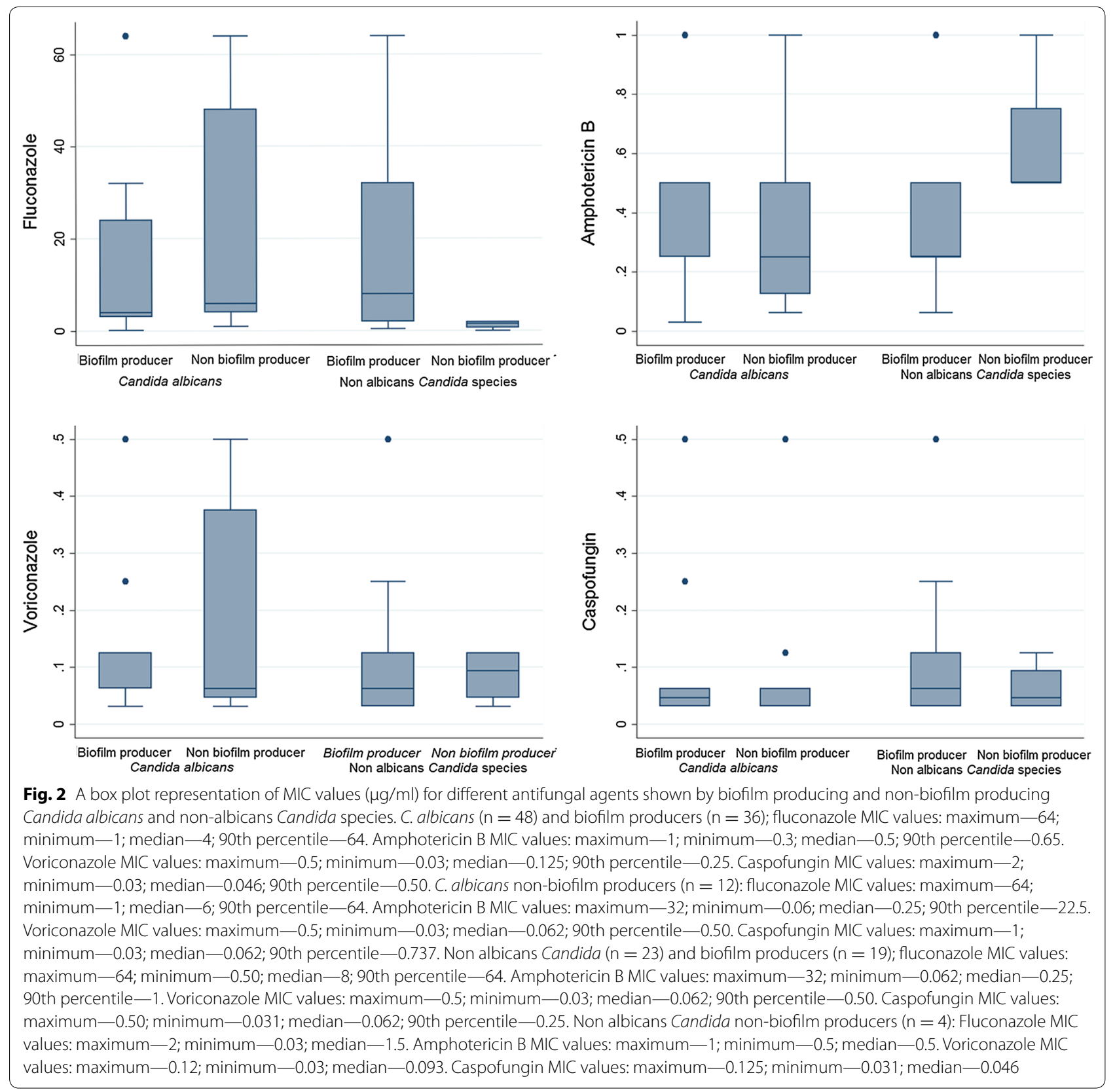

$31.7 \%$ of the Candida isolated from blood were resistant to fluconazole.

Apart from antifungal drug resistance in Candida, another major virulence attribute of this organism is production of biofilm that could lead to treatment failure and recurrence of infection. Tallaprageda et al. [19] noted high rate of biofilm production among the Candida isolates from blood stream and other invasive infections. Hassan et al. [22] found that significantly larger number of $C$. albicans isolates were biofilm producers as compared to the non-albicans Candida. While the exact reason for the higher rate of biofilm production among $C$. albicans was ill-understood, scanning electron microscopy studies of complex biofilm architectures attributed the integrity and strength of these biofilms to the higher number of hyphal elements produced by $C$. albicans than $C$. tropicalis and C. parapsilosis. The latter two species formed biofilms of lesser strength and the biofilm formed by these two species primarily consisted of micro colony aggregates of yeast cells [19]. In another recent investigation, Sariguzel et al. [11] detected biofilm among 33\% of non albicans Candida as compared to $25 \%$ of $C$. albicans. We also observed 
a comparatively higher rate of biofilm production among non albicans Candida as opposed to C. albicans (83\% vs. $75 \%$; Table 1). Notwithstanding the aforementioned variability in the rate of biofilm production among different Candida species, it is noteworthy that such high degree of biofilm forming ability among clinical Candida isolates reflects the potential of these organisms to cause invasive disease $[4,22]$. Thus, biofilm production could be a classic prototypical phenotypic marker of pathogenicity of a distinct population of Candida, differentiating these from mere commensals $[1,2,23]$.

We observed that non-biofilm producing C. albicans and non albicans Candida showed high MICs towards fluconazole, amphotericin B and voriconazole. This correlation, however, could not be detected among C. albicans isolates (Fig. 2). Unlike in bacterial pathogens [24], studies involving correlation between biofilm production and multidrug resistance among Candida are scanty [20, 25-27]. Our study, however, highlighted that majority of the non albicans Candida strains that were biofilm producers had shown high MICs towards fluconazole (Fig. 2).

\section{Conclusion}

Non-albicans Candida species are emerging as potential threats to cause invasive disease and posing a therapeutic challenge. Detection of high rate of biofilm activities among non-albicans Candida species along with high level of fluconazole resistance warrant wider surveillance of Candida isolates in order to clearly define the exact role of biofilms and drug resistance in invasive candidiasis.

\section{Limitations}

Non-albicans Candida isolates in our study were few. Thus in order to hypothesise that more of non-albicans Candida were capable of forming biofilms as compared to $C$. albicans, further studies, including higher numbers, would be required. Testing of hypothesis of inferential statistics was not applicable for this study because of the inadequate sample size.

\section{Additional file}

Additional file 1: Table S1. Isolation rates and percentage isolation of $C$. albicans vs. non-albicans Candida including their biofilm producing abilities in various clinical specimens.

\footnotetext{
Abbreviations

ATCC: American Type Culture Collection; BSA: bovine serum albumin; CFG: caspofungin; CFU: colony forming units; CLSI: Clinical and Laboratory Standards Institute; CSH: cell surface hydrophobicity; DNAse: deoxyribonuclease; MIC: minimum inhibitory concentration; PBS: phosphate buffered saline; SDA: sabouraud dextrose agar; Vs: versus; XTT: sodium 39-[1-(phenylaminocarbonyl)-3,4-tetrazolium]-bis(4-methoxy-6-nitro)benzene sulfonic acid hydrate.
}

\section{Authors' contributions}

SHS conceived and designed the study, collected specimens, undertook lytic compounds activity assay, performed the antifungal sensitivity testing, analysed data and wrote the manuscript. BPB, NKS and YM contributed towards identification of yeasts, lytic compounds activity assays and antifungal sensitivity testing and data analysis. BS assisted with the statistical analysis. NN contributed towards interpretation of the antifungal susceptibility test results and the manuscript writing and critical evaluation of manuscript. IB and SG contributed towards distilling the material and manuscript preparation. All authors read and approved the final manuscript.

\section{Author details}

${ }^{1}$ Manipal College of Medical Sciences, Pokhara, Nepal. ${ }^{2}$ Melaka Manipal Medical College, Manipal, India.

\section{Acknowledgements}

The authors gratefully acknowledge the faculty and technical staffs, department of microbiology for support. We extend our special thanks to Manipal Teaching Hospital, Pokhara, Nepal, for providing the facility to carry out the study.

Competing interests

The authors declare that they have no competing interests.

\section{Availability of data and materials}

The datasets used and/or analysed during the current study available from the corresponding author on reasonable request.

Consent for publication

Not applicable.

\section{Ethics approval and consent to participate}

The research proposal was approved by the Institutional Ethics Committee, Manipal Teaching Hospital, Pokhara, Nepal, and their permission was obtained to use the clinical isolates in this study.

\section{Funding}

The authors have not received any funding from any agency to support the work presented in this submission.

\section{Publisher's Note}

Springer Nature remains neutral with regard to jurisdictional claims in published maps and institutional affiliations.

Received: 5 May 2017 Accepted: 23 October 2017

Published online: 02 November 2017

\section{References}

1. Pfaller MA, Dickema DJ. Epidemiology of invasive candidiasis: a persistent public health problem. Cin Microbiol Rev. 2007;20:133-63.

2. Deepa $K$, Jeevitha T, Michael A. In vitro evaluation of virulence factors of Candida species isolated from oral cavity. J Microbiol Antimicrob. 2015;73:28-32.

3. Perlroth J, Choi B, Spellberg B. Nosocomial fungal infections: epidemiology, diagnosis and treatment. Med Mycol. 2007:45:321-46.

4. Chakrabarti A, Singh K, Das S. Changing face of nosocomial candidemia. Indian J Med Microbiol. 1999:17:160-6.

5. Chander J, Singla N, Sidhu SK, et al. Epidemiology of Candida blood stream infections: experience of a tertiary care centre in North India. J Infect Dev Ctries. 2013;7:670-5.

6. Melek I, Mustafa AA, Ayse NK, Erkan Y, Omer E, Suleyman D, Gonca D. Investigating virulence factors clinical Candida isolates in relation to atmospheric conditions and genotype. Turk J Med Sci. 2012;42:1476-83.

7. Subramanya SH, Sharan NK, Baral BP, et al. Diversity, in vitro virulence traits and antifungal susceptibility pattern of gastrointestinal yeast flora of healthy poultry, Gallus gallus domesticus. BMC Microbiology. 2017;17:113. https://doi.org/10.1186/s12866-017-1024-4. 
8. Staib F. Serum proteins as nitrogen source of yeast like fungi. Sabouraudia. 1965;4:187-93.

9. Samarnayake LP, Raeside JM, Mac Harlane TW. Factors affecting the phospholipase activity of Candida species in vitro. Sabouraudia. 1984;22:201-7.

10. Slifkin M. Tween 80 opacity test response of various Candida species. J Clin Microbiol. 2000;38:4626-8.

11. Ruan E, Kilian W, Hugo A, Albertyn J, Kock JLF, Pohl CH. Virulence of South African Candida albicans strains isolated from different clinical samples. Med Mycol. 2014;52:246-53.

12. CLSI. Reference method for broth dilution antifungal susceptibility testing of yeasts; approved standard-third edition; CLSI document M27-A3. Wayne: Clinical and Laboratory Standards Institute, 2008.

13. Sariguzel FM, Berk E, Koc AN, Sav H, Demir G. Investigation of the relationship between virulence factors and genotype of Candida spp. isolated from blood cultures. J Infect Dev Ctries. 2015;9:857-64.

14. Mohandas V, Ballal M. Proteinase and phospholipase activity as virulence factors in Candida species isolated from blood. Rev Iberoam Micol. 2008;25:208-10.

15. Sardi JC, Duque C, Hofling JF, Goncalves RB. Genetic and phenotypic evaluation of Candida albicans strains isolated from subgingival biofilm of diabetic patients with chronic periodontitis. Med Mycol. 2012;50:467-75.

16. Chakrabarti A, Nayak N, Talwar P. In vitro proteinase production by Candida Species. Mycopathologia. 1991;144:163-8.

17. Gokee G, Cerikcioglu N, Yaget A. Acid proteinase, phospholipase, and biofilm production of Candida species isolated from blood cultures. Mycopathologia. 2007;164:265-9.

18. Yucesoy M, Marol S. Determination of esterase activity of Candida varieties. Mikrobiyol Bul. 2003;37:59-63.

19. Tellapragada C, Eshwara VK, Johar R, Shaw T, Malik N, Bhat PV, Kamath A, Mukhopadhyay C. Antifungal susceptibility patterns, In vitro production of virulence factors, and evaluation of diagnostic modalities for the speciation of pathogenic Candida from blood stream infections and vulvovaginal candidiasis. J Pathog. 2014. https://doi.org/10.1155/2014/142864.
20. Deorukhkar SC, Saini S, Mathew S. Virulence factors contributing to pathogenicity of Candida tropicalis and its antifungal susceptibility profile. Int J Microbiol. 2014. https://doi.org/10.1155/2014/456878.

21. Seneviratne CJ, Rajan S, Wong SS, Tsang DNC, Lal CKC, Samaranayake $L P$, Jin L. Antifungal susceptibility in serum and virulence determinants of Candida blood stream isolates from Hong Kong. Front Microbiol. 2016;7:1-8.

22. Hassan F, Xess I, Wang $X$, Jain N, Fries BC. Biofilm formation in clinical Candida isolates and its association with virulence. Microbes Infection. 2009;11:753-61.

23. Bhatt M, Sarangi G, Paty BP, Mohapatra D, Chayani N, Mahapatra A, Das P, Sahoo D. Biofilm as a virulence marker in Candida species in nosocomial blood stream infections and its correlation with antifungal resistance. Indian J Med Microbiol. 2015;33:112-4.

24. Prasad S, Nayak N, Satpathy G, Nag HL, Venkatesh P, Ramakrishnan S, Ghose S, Nag TC. Molecular and phenotypic characterization of Staphylococcus epidermidis in implant related infections. Indian J Med Res. 2012:136:95-102.

25. De Luca C, Guglielminetti M, Ferrario A, Calabrio M, Casari E. Candidemia: species involved, virulence factors and antimycotic susceptibility. New Microbiol. 2012;35:459-68.

26. Tosun I, Akyuz Z, Guler NC, Gulmez D, Bayramoglu G, Kaklikkaya N, Arikan-Akdagli S, Aydin F. Distribution, virulence attributes and antifungal susceptibility patterns of Candida parapsilosis complex strains isolated from clinical samples. Med Mycol. 2013;51:483-92.

27. Anwar H, Strap JL, Costerton W. Establishment of aging biofilms: possible mechanism of bacterial resistance to antimicrobial therapy. Antimicrob Agents Chemother. 1992;36:1347-51.

\section{Submit your next manuscript to BioMed Central and we will help you at every step:}

- We accept pre-submission inquiries

- Our selector tool helps you to find the most relevant journal

- We provide round the clock customer support

- Convenient online submission

- Thorough peer review

- Inclusion in PubMed and all major indexing services

- Maximum visibility for your research

Submit your manuscript at www.biomedcentral.com/submit
O Biomed Central 